\title{
AC 2008-2183: ONLINE, INTRODUCTORY MICROCONTROLLER LABS AND EXERCISES FOR ENGINEERING STUDENTS
}

\section{Marc McComb, Microchip Technology, Inc.}

As Technical Training Engineer with Microchip's Security, Microcontroller \& Technology Development Division, Marc McComb develops educational materials for customers on Microchip's 8-bit PIC® Microcontrollers (MCUs). These materials take the form of Regional Training Center (RTC) classes, Web seminars and lab exercises for the Company's Academic Program. Prior to this position, Marc worked for 13 years as a paramedic in Ontario, Canada until a back injury forced a career change. He returned to school and graduated from St. Lawrence College's Electronics Engineering Technology program in Kingston, Ontario, Canada in 2006. Marc is married and has two teen-aged step children.

\#\#\#

The Microchip name and logo, and PIC are registered trademarks of Microchip Technology Inc. in the U.S.A. and other countries. All other trademarks mentioned herein are property of their respective companies. 


\title{
Online, Introductory Microcontroller Labs and Exercises for Engineering Students
}

\begin{abstract}
This paper will explain several introductory, hands-on labs developed for Web-based teaching and learning. Utilizing the Internet to deliver self-directed, inexpensive, current instructional material extends the role of educational institutions. With instant availability, low cost, convenience and better accessibility, online education increases the pool of students that can be reached.

The labs presented here are based upon Microchip Technology's 8-bit PIC ${ }^{\circledR}$ microcontroller (MCU) architecture, as well as its peripherals and possible applications. Only the most fundamental of embedded knowledge is assumed, such as knowledge of binary numbering systems and basic digital circuitry. The labs could potentially serve as a resource for university/college professors who would like to use them in the classroom.

This paper will explain how these labs are formatted, how to use them and the learning objectives or benefits to the student that each provides. One lab will be chosen and dissected, to provide the audience with an insightful overview of the general format used. Future labs will also be discussed, to provide the audience with a clear understanding of the direction in which this program is moving.
\end{abstract}

\section{Introduction}

The Internet provides an opportunity for both educators and employers to offer quality educational resources for engineering students. Introductory learning materials developed by a manufacturer can supply students with unique insight into a technology or process that complements what is learned in the classroom. Microchip's Academic Program has recently dedicated a section of its Web site to such learning resources. There, the student will find introductory materials on 8bit PIC MCUs and related topics. These materials include introductory tutorials, presentations, labs and exercises on the basics of architecture, peripherals, methods to optimize design using the PIC MCU and common applications. The intention is to provide the student with a solid foundation in embedded technology that will serve as a stepping stone into the more advanced topics that are covered in application notes and technical briefs provided by Microchip and other manufacturers. These labs have been formatted so that they can be easily implemented into a classroom environment by a professor, or used by the student as supplementary information to embedded concepts learned in university/college engineering curriculum. 


\section{Assessment of Learning Materials}

Learning materials undergo a rigorous review process before being made available to the Web site. First, all labs undergo a "dry run," where test subjects are asked to complete each lab and then provide a written assessment of lab content. Test subjects are selected from a wide range of backgrounds including engineering, new engineering graduates, technical writers and even interns from within the company. Following this dry run, labs are then sent on to a review process wherein a number of senior-level engineers are asked to review the material and offer comments, to ensure technical accuracy and that learning objectives have been met. This review process is two-fold - the original draft is submitted for the first round of comments. Once the comments have been incorporated into the original draft, a final draft is submitted for a final review. Any learning material will only be posted on the Web site once this review process is complete and the material has been deemed technically accurate and intuitive.

\section{Tools Needed to Utilize Learning Materials}

All of the resources required to complete the labs, tutorials and exercises are provided free of charge. A current, free version of Microchip's MPLAB ${ }^{\circledR}$ Integrated Development Environment (IDE) and the datasheets for applicable PIC MCUs are all easily accessible from www.microchip.com. Neither the student nor the professor needs to purchase any development tools or Silicon, as all labs, tutorials and exercises can be easily completed using the MPLAB SIM simulator. Students and educators have an opportunity to become familiar with these tools, so they can make an informed decision should they choose to purchase any products. Furthermore, any products purchased through Microchip's Academic Program come at a reduced price. Each lab includes a list of prerequisites that the user should have to fully benefit from the content. In general, labs are developed for the $\mathrm{C}$ programming language. The document entitled "Introduction to MPLAB IDE/PICC-Lite ${ }^{\text {TM }}$ Compiler Tutorial" (DS41322A) on Microchip's Web site is a typical prerequisite. This document provides download instructions for both the MPLAB IDE and the free PICC-Lite Compiler, along with a preparatory tutorial on using the tools and the simulator. 


\section{Example Lab}

The first lab posted on Microchip's Web site introduces students to a simple Timer/Counter peripheral, Timer0, and the relevant mid-range PIC MCUs. The objectives of this lab are to provide an understanding of the main components and the configuration of peripheral registers for Timer0, to produce a working firmware application. The lab assumes a basic understanding of circuit theory, basic digital electronics such as gates and registers, binary numbering systems and some programming experience using the $\mathrm{C}$ language. Students should complete the "Introduction to MPLAB IDE/PICC-Lite" Compiler tutorial to benefit fully from this lab. Again, the only resources required are the MPLAB IDE, the PICCLite compiler and the datasheet for the PIC16F690 MCU. All are available free of charge from www.microchip.com. This lab is the first in a two-part series on the Timer0 peripheral. The second lab uses the same application, only it uses Timer0 interrupts rather than the polling technique to develop a more robust design.

\section{Lab Synopsis}

This lab begins with an introduction to Timer/Counter peripherals. The difference between a Timer and a Counter are discussed, along with examples of basic implementations. Next, each of the main components that may be found on a given Timer/Counter module produced by Microchip—such as the source clock, Prescaler, Postscaler and Timer value register-is discussed as to how it affects the operational characteristics of the peripheral as a whole.

Each Timer0 configuration bit is described by location and its effect on the overall operational characteristics of the peripheral. Diagrams similar to those found in the datasheet are used for cohesion, only they are discussed in greater detail.

\section{“Hands-On" Lab}

To reinforce the functional characteristics of the Timer0 peripheral, a hands-on lab is next, which is introduced using the MPLAB IDE/PICC-Lite compiler. The student is directed to develop a new project file and generate a .c source file using code provided in the lab document. Using the provided code, students have functioning, completed firmware, allowing them to focus their attentions on simulating the application rather than writing code - at this point. The firmware application increments an 8-bit counter variable by one, whenever the 8-bit Timer0 value register overflows from 255 to 0 . A feature of the MPLAB SIM debugger called the Watch Window is used to track changes made to selected registers. The student adds to this Watch Window the register containing the current Timer0 value (TMR0), the register containing the Timer0 overflow flag (INTCON), the OPTION register (which contains the Timer0 peripheral configuration bits) and, finally, the counter variable to be incremented. 
The first part of the lab instructs the student to step through each line of code and observe the effects on the registers, as shown in the Watch Window. Students are to confirm that the counter variable does increment only on a TMR0 register overflow. Next, the debugger's Stopwatch feature is used to assess the timing characteristics of the application firmware. As each feature is introduced, basic operation and configuration is discussed. The student is to confirm that the TMR0 register increments 1:1 with the PIC MCU's internal instruction clock, using the Stopwatch. An equation used to determine the internal instruction clock-cycle period is discussed and then expanded upon, to determine the time to a TMR0 overflow. Next, the effect of writing to the TMR0 register is dissected and implemented in the provided firmware.

\section{Exercises}

After completing the lab, students should be familiar enough with the TMR0 peripheral and the MPLAB IDE tools to begin basic firmware modifications. Exercises are then introduced to reinforce concepts learned. Answers are not provided in the document itself, and can be made available to professors only or to the student as well. The Timer0 Lab exercises challenge the student to develop firmware using the prescaler, and then simulate the result using the debugger tools discussed. Next, the student is asked to manipulate the equations discussed, to accommodate for the prescaler. Finally, the student is to increment the counter variable in 4 millisecond $(\mathrm{mS})$ increments.

\section{Measuring Learning Objectives}

The exercises at the end of each lab are intended to measure whether learning objectives have been met, and to what extent. An online lab evaluation survey has also been provided, so that students and professors can supply feedback to lab developers. This evaluation form has been formatted so that the responder can provide essay-style feedback, and verbose responses are encouraged. In this way, current and future labs can be improved upon to ensure an intuitive and worthwhile learning experience.

\section{Summary}

The intentions- of these labs are to not only provide an education on the peripheral or topic at hand, but also to assist students in developing analytical skills as they relate to embedded design, through the use of the simulator and other MPLAB IDE tools. This program is expected to evolve, in order to remain current as technological trends change. Topics beyond the PIC MCU architecture and peripherals, such as embedded software techniques, mixed-signal design and common applications, are expected to be offered, to provide a complete learning experience.

\section{\#\#\#}

Note: The Microchip name and logo, MPLAB and PIC are registered trademarks of Microchip Technology Inc. in the U.S.A. and other countries. All trademarks mentioned herein are property of their respective companies. 